Joachim Drevs

Integrative Onkologie 



\section{Joachim Drevs}

\section{Integrative Onkologie}

Definition - Inhalte - Bedeutung

\section{DE GRUYTER}


Autor

Prof. Dr. med. Joachim Drevs

UNIFONTIS

Praxisklinik für Integrative Onkologie

Apothekenweg 6

38173 Sickte

ISBN: 978-3-11-049648-2

e-ISBN (PDF): 978-3-11-049710-6

e-ISBN (EPUB): 978-3-11-049373-3

Library of Congress Control Number: 2019932020

Bibliografische Information der Deutschen Nationalbibliothek

Die Deutsche Nationalbibliothek verzeichnet diese Publikation in der Deutschen Nationalbibliographie; detaillierte bibliografische Daten sind im Internet über http://dnb.d-nb.de abrufbar.

Der Verlag hat für die Wiedergabe aller in diesem Buch enthaltenen Informationen mit den Autoren große Mühe darauf verwandt, diese Angaben genau entsprechend dem Wissensstand bei Fertigstellung des Werkes abzudrucken. Trotz sorgfältiger Manuskriptherstellung und Korrektur des Satzes können Fehler nicht ganz ausgeschlossen werden. Autoren und Verlag übernehmen infolgedessen keine Verantwortung und keine daraus folgende oder sonstige Haftung, die auf irgendeine Art aus der Benutzung der in dem Werk enthaltenen Informationen oder Teilen davon entsteht.

Die Wiedergabe der Gebrauchsnamen, Handelsnamen, Warenbezeichnungen und dergleichen in diesem Buch berechtigt nicht zu der Annahme, dass solche Namen ohne weiteres von jedermann benutzt werden dürfen. Vielmehr handelt es sich häufig um gesetzlich geschützte, eingetragene Warenzeichen, auch wenn sie nicht eigens als solche gekennzeichnet sind.

(C) 2020 Walter de Gruyter GmbH, Berlin/Boston

Einbandabbildung: udra/iStock/gettyimages/

Satz/Datenkonvertierung: L42 AG, Berlin

Druck und Bindung: CPI Books GmbH, Leck

www.degruyter.com 
Mit besonderem Dank an

Leona Kröhle

Für die Unterstützung in der

schwierigen Zeit der Entstehung des Buches 
